Proc. XII Int. School on Theoretical Physics — Symmetry and Structural Properties of Condensed Matter

\title{
Anomalous Magnetocaloric Properties of the Spin-1/2 Ising Model on a Decorated Square Lattice in a Vicinity of Second-Order Phase Transition
}

\begin{abstract}
K. KarL’OVÁ* AND J. StreČKA
Institute of Physics, Faculty of Science, P.J. Šafárik University, Park Angelinum 9, 04001 Košice, Slovakia

Magnetocaloric properties of the spin-1/2 Ising model on a decorated square lattice in a transverse magnetic field are investigated by the use of a generalized decoration-iteration transformation, which establishes a rigorous mapping correspondence with the zero-field spin- $1 / 2$ Ising model on a square lattice. The temperature dependence of the entropy, the isothermal entropy change and the adiabatic temperature change display anomalous singular behavior in a vicinity of a second-order phase transition. The large inverse magnetocaloric effect can be found in the isothermal entropy change within the temperature interval, which is delimited by the critical temperatures at zero and non-zero transverse fields.
\end{abstract}

DOI: 10.12693/APhysPolA.132.170

PACS/topics: 75.10.Hk, 75.30.Sg, 75.40.-s, 75.50.Cc

\section{Introduction}

The magnetocaloric effect (MCE) refers to the heating or cooling of a magnetic material when subjected to a variation of magnetic field. The phenomenon of MCE was firstly observed by Warburg in 1881 [1]. MCE can be characterized by means of the isothermal magnetic entropy change $\Delta S_{i s o}$, which occurs when the magnetic field is applied isothermally [2]. A significance of the MCE goes with its potential utilization for a magnetic refrigeration through the process of adiabatic demagnetization [3]. The magnetic materials with the large MCE are therefore of technological interest with regard to their cooling capability either at room or low temperatures [4]. An enhanced MCE can be normally observed in the vicinity of the phase transitions [5].

In the present work we will examine the magnetocaloric properties, namely, the entropy as a function of temperature, the isothermal entropy change and the transversefield dependence of the temperature of the spin- $1 / 2$ Ising model on a decorated square lattice, which is one of the few exactly solvable two-dimensional models exhibiting a second-order phase transition.

\section{Model and method}

Let us consider the spin- $1 / 2$ Ising model on a decorated square lattice in a transverse magnetic field (see Fig. 1), which can be defined through the following Hamiltonian:

$$
\hat{\mathcal{H}}_{\mathrm{d}}=-J \sum_{\langle i, k\rangle}^{4 N} \hat{S}_{i}^{z} \mu_{k}^{z}-\Omega \sum_{i=1}^{2 N} \hat{S}_{i}^{x}
$$

where $\mu_{k}^{z}= \pm 1 / 2$ represent the nodal Ising spins, $\hat{S}_{i}^{z}$

\footnotetext{
* corresponding author; e-mail: katarina.karlova@student.upjs.sk
}

and $\hat{S}_{i}^{x}$ represent the relevant spatial components of the standard spin- $1 / 2$ operator of the decorating atoms. The parameter $J$ is the Ising interaction between the nearestneighbor nodal and decorating spins, while the second term $\Omega$ accounts for Zeeman's energy of the decorating atoms in a transverse magnetic field. Finally, $N$ denotes the total number of atoms of the original lattice.

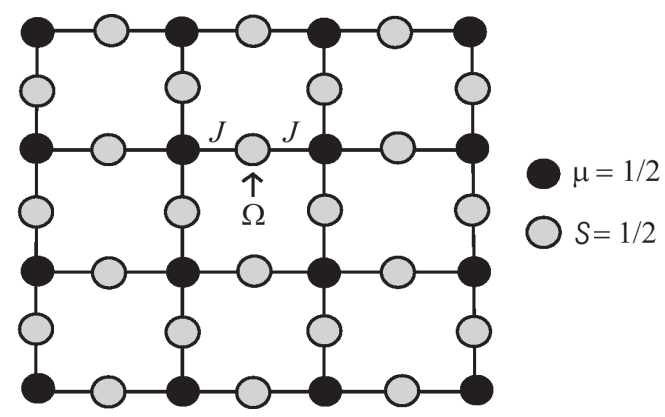

Fig. 1. A part of the decorated square lattice. The black circles denote lattice position of the nodal spin$1 / 2$ atoms and the gray circles schematically represent lattice position of the spin- $1 / 2$ decorating atoms.

The phase diagram, thermodynamics, longitudinal and transverse magnetizations of the transverse Ising model on a decorated square lattice defined through the Hamiltoninan (1) was comprehensively studied by the use of a generalized decoration-iteration transformation, which establishes a precise mapping equivalence with an effective spin-1/2 Ising model on a simple square lattice with the know exact solution due to Onsager [6]. From the rigorous mapping correspondence one may consequently derive exact expressions for all physical quantities of interest for the transverse Ising model on a decorated square lattice as well. The readers interested in detail of relevant calculation procedure are referred to Refs. [7, 8]. In the present work we will focus our attention on magnetocaloric properties, specifically on MCE close to the 
second-order phase transition. The entropy as a function of temperature can be obtained from Eq. (8) of Ref. [8] and the isothermal entropy change can be calculated as a difference of the entropy at non-zero and zero magnetic field $\Delta S_{i s o}=S_{2}(T, \Omega \neq 0)-S_{1}(T, \Omega=0)$ at the constant temperature.

\section{Results and discussion}

Let us start our discussion with typical temperature dependences of the molar entropy per one spin $S_{d}\left(\mathrm{~J} \mathrm{~K}^{-1} \mathrm{~mol}^{-1}\right)$, which are displayed in Fig. 2 for a few different values of the transverse magnetic field. The entropy monotonously increases with increasing temperature at zero as well as non-zero transverse fields, whereas the most pronounced changes can be found close to a second-order phase transition point accompanied with a weak singular behavior of the type $\left(T-T_{c}\right) \ln \left|T-T_{c}\right|$ (open circles in Fig. 2). The temperature, at which the curve at non-zero transverse field crosses the zero-field curve (red solid line) represents a crossover temperature, since it determines the change between the conventional and inverse MCE. At higher transverse fields $(\Omega / J \geq 1.0)$ the entropy is higher than the zero-field entropy below the crossover temperature. Contrary to this, the entropy at higher transverse fields is lower than the zero-field entropy above the crossover temperature. It is worthwhile to remark that the entropy reaches at high transverse fields (e.g. $\Omega / J=4.0$ ) a constant value $S_{d}=R_{m} 1 / 3 \ln 2 \approx 1.921 \mathrm{~J} \mathrm{~K}^{-1} \mathrm{~mol}^{-1}$ ( $R_{m}$ is universal gas constant), which is in agreement with the disordered nature of the nodal Ising spins and the full alignment of the decorating spins into the transverse magnetic field. On the other hand, two crossover temperatures can be detected in Fig. 2 at lower transverse fields like for instance $\Omega / J=0.5$. The entropy is lower than the zero-field entropy below the first crossover temperature $k_{\mathrm{B}} T / J \approx 0.26$ and above the second crossover temperature $k_{\mathrm{B}} T / J \approx 0.33$, while it becomes higher within the temperature range in between both crossover temperatures. Owing to this fact, one observes a double transition between the conventional and inverse MCE.

MCE can be even better characterized through the isothermal entropy change $-\Delta S_{i s o}$ in response to a variation of the applied transverse field. Temperature dependence of the isothermal temperature change is shown in Fig. 3 at three different values of the transverse-field change $\Delta \Omega$. The crosses on relevant isothermal curves mark weak singularities of the zero-field entropy of the type $\left(T-T_{c}\right) \ln \left|T-T_{c}\right|$ occurring at the critical temperature $k_{\mathrm{B}} T_{c} / J \approx 0.327$, while open circles denote similar weak singularities of the entropy located at critical points of second-order phase transitions at the upper transverse fields $\Omega / J=0.5,1.0$, and 2.0 , respectively. The positive isothermal entropy change $-\Delta S_{\text {iso }}>0$ implies the conventional $\mathrm{MCE}$, while the negative isothermal entropy change $-\Delta S_{\text {iso }}<0$ serves in evidence of the inverse MCE. It can be seen from Fig. 3 that the large inverse

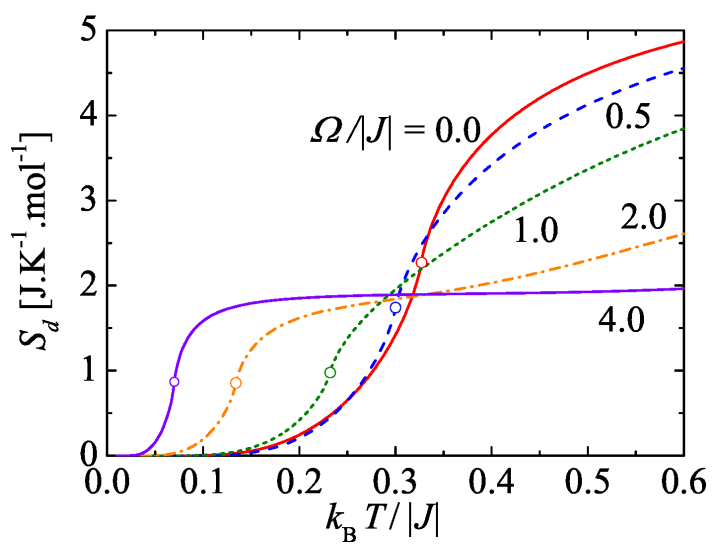

Fig. 2. The molar entropy as a function of temperature at a few values of the transverse magnetic field.

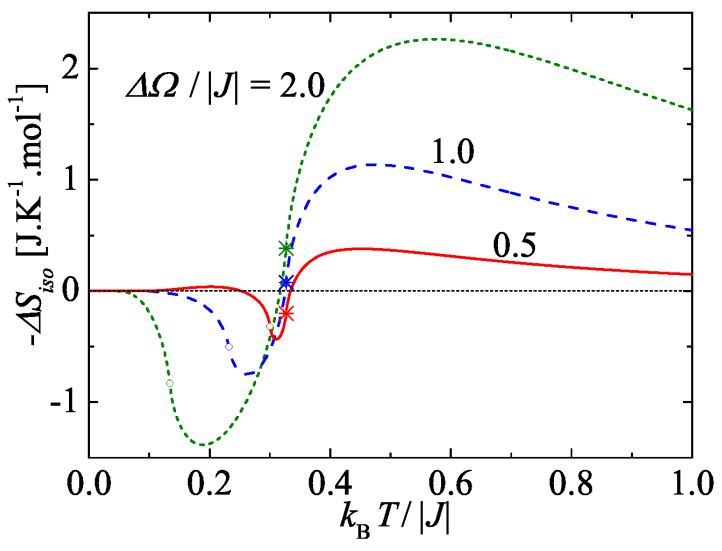

Fig. 3. The temperature dependence of the isothermal entropy change at three different values of the transverse-field change. Thin dotted line at $-\Delta S_{\text {iso }}=0$ is guide for eyes only.

MCE occurs within the temperature interval $T_{c}(h \neq 0)<$ $T<T_{c}(h=0)$, which is delimited by both critical temperatures. The inverse MCE gradually increases and shifts to lower temperatures upon strengthening of the transverse-field change $\Delta \Omega / J$. While the inverse MCE initially dominates over the low-temperature region at higher changes of the transverse field $(\Delta \Omega / J=1.0$ and $2.0)$, the smaller transverse-field change $\Delta \Omega / J=0.5$ leads to the small conventional MCE at relatively low temperatures below the crossover temperature in accordance with Fig. 2. On the other hand, one can observe a maximum of the conventional MCE in a hightemperature region bounded from below by a zero-field critical temperature, whereas its height and locus increases with increase of the transverse field.

Another important characteristics of MCE represents an isentropic change of temperature induced upon variation of the transverse magnetic field, which is displayed in Fig. 4. As one can see, there is a highest density of isentropic lines around the line of second-order phase transitions, which is depicted in Fig. 4 as thick broken line. This result is in accordance with the aforementioned 


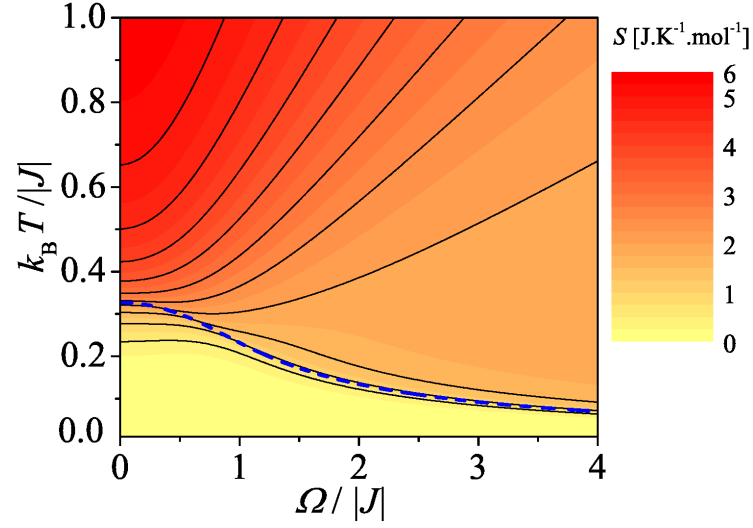

Fig. 4. A density plot of the molar entropy in the field-temperature plane. The broken line indicates monotonous decline of the critical temperature with increase of the transverse field.

behavior of the entropy discussed at Fig. 2. It can be observed from Fig. 4 that the temperature rapidly decreases during the process of the adiabatic demagnetization at relatively high entropies until it tends towards some constant finite value. On the other hand, the temperature increases with decrease of transverse field at relatively low entropies.

\section{Conclusion}

In this work, we have investigated the magnetocaloric properties of the exactly solvable spin- $1 / 2$ Ising model on a decorated square lattice in a transverse magnetic field. The temperature dependences of the entropy have been examined for a few different values of the transverse magnetic field. The MCE was demonstrated either through the positive or negative isothermal entropy change. It has been evidenced that the large inverse MCE accompanied with the negative isothermal entropy change $-\Delta S_{i s o}$ emerges in a vicinity of the second-order phase transitions. Another important aspect of MCE represents the transverse-field dependence of the temperature under the adiabatic condition. The most dense isentropes are situated in a close vicinity of the continuous phase transitions.

\section{Acknowledgments}

This work was financially supported by Ministry of Education, Science, Research and Sport of the Slovak Republic provided under the grant No. VEGA 1/0331/15, by the grant of Slovak Research and Development Agency under the contract No. APVV-14-0073 and the internal grant of Faculty of Science of P.J. Šafárik University under the contract No. VVGS-PF-2016-72606.

\section{References}

[1] E. Warburg, Ann. Phys. 249, 141 (1881).

[2] V.K. Pecharsky, K.A. Gschneidner, J. Magn. Magn. Mater. 200, 44 (1999).

[3] Y. Spichkin, A.K. Zvezdin, S.P. Gubin, A.S. Mischenko, A.M. Tishin, J. Phys. D Appl. Phys. 34, 1162 (2001).

[4] P.J. von Ranke, M.A. Mota, D.F. Grangeia, A.M.G. Carvalho, F.C.G. Gandra, A.A. Coelho, A. Caldas, N.A. de Oliveira, S. Gama, Phys. Rev. B 70, 134428 (2004).

[5] A.M. Tishin, Y.I. Spichkin, The Magnetocaloric Effect and Its Applications, IOP Pub., Bristol 2003.

[6] L. Onsager, Phys. Rev. 65, 117 (1944).

[7] M. Jaščur, J. Strečka, Phys. Lett. A 258, 47 (1999).

[8] J. Strečka, M. Jaščur, J. Magn. Magn. Mater. 260, 415 (2003). 